\title{
Digitales Datenmanagement als Berufsfeld im Kontext der Data Literacy
}

\section{Digital Data Management - a Professional Field in the Data Literacy Context}

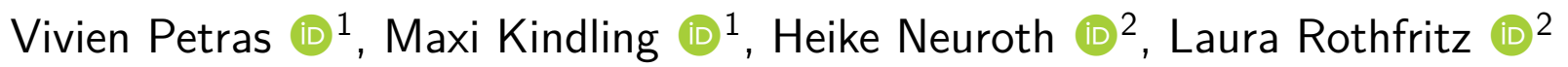 \\ ${ }^{1}$ Institut für Bibliotheks- und Informationswissenschaft \\ Humboldt-Universität zu Berlin \\ Dorotheenstr. 26, 10117 Berlin \\ vivien.petras@ibi.hu-berlin.de / maxi.kindling@ibi.hu-berlin.de \\ ${ }^{2}$ Fachbereich Informationswissenschaften \\ Fachhochschule Potsdam \\ Kiepenheuerallee 5, 14469 Potsdam \\ neuroth@fh-potsdam.de / laura.rothfritz@fh-potsdam.de
}

\begin{abstract}
Deutsch
Das Institut für Bibliotheks- und Informationswissenschaft der Humboldt-Universität zu Berlin und der Fachbereich Informationswissenschaften der Fachhochschule Potsdam entwickeln den weiterbildenden Masterstudiengang Digitales Datenmanagement. Er vermittelt Kompetenzen der Forschungs- und Handlungsfelder im Datenmanagement unter Berücksichtigung der nationalen und internationalen wissenschaftspolitischen, organisatorischen, technischen und rechtlichen Rahmenbedingungen. Im Mittelpunkt steht die Vermittlung von Fähigkeiten im analytischen wie praktischen Umgang mit Forschungs- und Informationsinfrastrukturen besonders in der Domäne Wissenschaft und Forschung sowie der Ermittlung von Anforderungen und Lösungen für Informationssysteme, die das Datenmanagement unterstützen.
\end{abstract}




\begin{abstract}
English
The Berlin School for Library and Information Science at Humboldt-Universität zu Berlin and the Faculty of Information Sciences at the University of Applied Sciences Potsdam develop the executive Master's study program Digital Data Management. It provides competencies in the research and activity areas of data management while considering national and international science policies as well as organizational, technical and legal contexts. The educational focus is on capabilities in analytic and practical processing in research and information infrastructures, particularly in the academic and research domains, and requirements analysis and solutions for information systems, which support data management.
\end{abstract}

Schlüsselwörter: Digitales Datenmanagement, Data Literacy, Data Stewardship, Informationsinfrastrukturen, Forschungsdaten

Keywords: Digital Data Management, Data Literacy, Data Stewardship, Information infrastructures, Research data 


\section{Einführung}

Datenmanagement ist neben Data Science ein wichtiger Teilbereich der Data Literacy. Sie wird in aktuellen, einschlägigen wissenschaftspolitischen Positionspapieren als ein Berufsfeld beschrieben, für das zukünftig hohe Investitionen geplant sind.

Die Hochschulrektorenkonferenz (HRK) schreibt speziell zu Forschungsdaten in 2016: „Forschungsdaten sind ein wertvolles Gut mit großen Potenzialen für Wissenschaft, Gesellschaft und Wirtschaft." Hochschulen können „mit ihrem Studienangebot die neuen beruflichen Profile schaffen, die für die Etablierung und den Betrieb des Forschungsdatenmanagements unentbehrlich sind."

Auch der Rat für Informationsinfrastrukturen (RfII) nimmt in seinen Empfehlungen zum Forschungsdatenmanagement in Deutschland an das Bundesministerium für Bildung und Forschung (BMBF) neben wichtigen Anforderungen an Infrastruktur und Fördermechanismen besonders die Personalentwicklung in den Fokus:

Datenintensive Forschung wie auch Lehre benötigen einschlägig qualifizierte Personen - und dies in großer Zahl. Hier läuft der Arbeitskräftemarkt in Deutschland der globalen Entwicklung in gefahrvoller Weise hinterher. Daher gilt es aus Sicht des RfII, schnellstmöglich kompetente Forschergenerationen und spezialisierte Fachkräfte für neue Berufsbilder im Bereich des Datenmanagements auszubilden. ${ }^{2}$

Auf europäischer Ebene identifizierte eine von der Europäischen Kommission eingesetzte High Level Expert Group im Zusammenhang mit der Entwicklung der European Open Science Cloud (EOSC) den Mangel an Experten im Umfeld des Datenmanagements als eine bedeutende Herausforderung. Ihre Aus- und Weiterbildung wird als ein Kernaspekt der EOSC benannt: „Core data experts need to be trained and their career perspective significantly improved."3

Dem Desiderat, Studienangebote zu schaffen, die für das Berufsfeld des Datenmanagements qualifizieren, sind das Institut für Bibliotheks- und Informationswissenschaft der Humboldt-Universität zu Berlin (IBI) und der Fachbereich Informationswissenschaften der Fachhochschule Potsdam (FB Informationswissenschaften) gefolgt. Im Rahmen einer für Deutschland einzigartigen Kooperation

1. Hochschulrektorenkonferenz, Gemeinsame Erklärung von Hochschulleitungen, die am Workshop der HRK zum Forschungsdatenmanagement am 16.12.2016 teilgenommen haben (2016).

2. RFII - Rat für Informationsinfrastrukturen, Leistung aus Vielfalt: Empfehlungen zu Strukturen, Prozessen und Finanzierung des Forschungsdatenmanagements in Deutschland (Göttingen, 2016), 3.

3. High Level Expert Group on the European Open Science Cloud, Realising the European Open Science Cloud (2016), 7. 
zweier bibliotheks- und informationswissenschaftlicher Institute entwickelten Kolleginnen ${ }^{4}$ beider Institute länder- und organisationsübergreifend den weiterbildenden Masterstudiengang Digitales Datenmanagement (DDM). Der Start des Studiengangs ist für das Sommersemester 2020 geplant.

Dieser Artikel beschreibt das Rahmenwerk, auf denen die Inhalte des Studienganges basieren sowie die Struktur und Organisation des Studiengangs. Ein weiterer Fokus wird auf die Voraussetzungen für am Studium Interessierte und die Potenziale gelegt, die sich aus dem Abschluss ergeben. Für eine ausführlichere Darstellung des inhaltlichen Hintergrunds und die Einbettung in aktuelle internationale sowie nationale Diskussionen und Entwicklungen sei auf einen weiteren Artikel in Bibliothek, Forschung und Praxis verwiesen. ${ }^{5}$

\section{Anforderungen an das Datenmanagement - Die Entwicklung eines Studiengangs}

Das EDISON Data Science Competences Framework ist das Resultat einer zweijährigen europäischen Studie, um Angebote und Anforderungen an Data-Science-Kompetenzen und -Fähigkeiten in Forschung und Wirtschaft zu eruieren. Das multinationale EDISON-Projekt ${ }^{6}$ hat die Definition und Standardisierung eines Berufsbildes für Data Science Professionals zum Ziel. In diesem Framework wird der Begriff Data Science als übergeordnete Bezeichnung verwendet, das fünf Kompetenzgruppen ${ }^{7}$ umfasst und die entsprechenden Fähigkeiten und Kenntnisse für diese Gruppen definiert: Data Analytics (Datenanalyse und Statistik), Data Science Engineering (Implementierung von Datenanalyseanwendungen), Data Management (Datenmanagement), Research Methods and Project Management (Forschungsmethoden und Projektmanagement) und Domain Related Competences (Domänenspezifische Kenntnisse).

Auch das Projekt EOSCpilot ${ }^{8}$ hat in Bezug auf die European Open Science Cloud unter anderem zum Ziel, ein Framework für erforderliche Kompetenzen und Fähigkeiten für das Datenmanagement zu entwickeln. Diesem liegen auch die Ergebnisse des EDISON Framework zugrunde. In den

4. Aus Gründen besserer Lesbarkeit wird nur das Femininum verwendet. Selbstverständlich sind Personen jeden Geschlechts angesprochen.

5. Heike Neuroth et al., „Digitales Datenmanagement als neue Aufgabe für wissenschaftliche Bibliotheken,“ erscheint, Bibliothek Forschung und Praxis.

6. http://edison-project.eu/ (18.01.2019)

7. Edison Project, EDISON Data Science Framework: Part 1. Data Science Competence Framework (CF DS). Release 2, 2017.

8. https://eoscpilot.eu/node (18.01.2019) 
EOSCpilot Reports D7. $1^{9}$ und D7. ${ }^{10}$ wurden die Kompetenzgruppen anhand des Lebenszyklus von Forschungsdaten angeordnet und ganzheitlich im Hinblick auf eine Unterscheidung der jeweiligen Stakeholder als "Service Users" und "Service Operators" beschrieben. ${ }^{11}$ Beide Projekte bieten wichtige Grundlagen für die Ausgestaltung der Studieninhalte des Masterstudiengangs Digitales Datenmanagement.

Die Bibliotheks- und Informationswissenschaft erforscht Aspekte des Datenmanagements (auch wenn man diese nicht immer mit dieser Terminologie beschreibt) und ist damit prädestiniert, wichtige Kompetenzen im Datenmanagement in der Lehre zu vermitteln. Ein Blick in die definierten Kompetenzen im EDISON Framework macht dies sehr deutlich. Für die DatenmanagementKompetenzgruppe wurden folgende Kompetenzen und Fähigkeiten definiert (zitiert aus dem englischen Original) ${ }^{12}$ :

○ Entwicklung und Implementierung von Datenmanagementstrategien für die Datensammlung, -speicherung, -archivierung und Zugänglichkeit für die weitere Verarbeitung,

- Entwicklung und Implementierung von Datenstrategien, insbesondere in Form von Datenmanagementpolicies und Datenmanagementplänen (DMP),

- Entwicklung und Implementierung von relevanten Datenmodellen, Definition von Metadaten mithilfe von Standards und etablierter Praktiken für verschiedene Datenquellen in verschiedenen wissenschaftlichen und wirtschaftlichen Domänen,

- Integration von heterogenen Daten von verschiedenen Quellen und Bereitstellung für weitere Analysen und Nutzung,

- Pflege von historischen Informationen zur Datenverarbeitung, einschließlich von Referenzen zu publizierten Daten und entsprechenden Datenquellen (Datenprovenienz),

○ Sicherstellung von Datenqualität, Zugänglichkeit, Interoperabilität, Standardkonformität und Publikation (Datenkuratierung),

- Entwicklung und Management / Kontrolle von Richtlinien für die Datensicherheit, Datenschutz, Urheberrecht und ethischen Grundsätzen im Datenmanagement.

9. Angus Whyte et al., Skills landscape analysis and competence model 7.1 (EOSCpilot, 2017)

10. Angus Whyte et al., Skills and Capability Framework Deliverable 7.3 (EOSCpilot, 2018)

11. Vgl. z.B. https://www.ianus-fdz.de/it-empfehlungen/lebenszyklus (18.01.2019)

12. Edison Project, EDISON Data Science Framework: Part 1. Data Science Competence Framework (CF DS). Release 2, 2017, 16, Table 4.1 
Die Bibliotheks- und Informationswissenschaft hat diese Kompetenzen unter Berücksichtigung spezieller institutioneller (z.B. in Bibliotheken u.a. Informationsinfrastruktureinrichtungen) bzw. organisatorischer, technischer und rechtlicher Bedingungen (z.B. unter Bewahrung von OpenScience-Prinzipien) traditionell im Fokus. Das, was heute unter dem Begriff „Data Literacy“13 als Schwerpunkt Datenmanagement beschrieben wird, ist bereits seit einigen Jahren Inhalt der Forschung und Lehre am IBI und am FB Informationswissenschaften. ${ }^{14}$

Wir verstehen Data Literacy als die Kompetenz des kritischen und lösungsorientierten Umgangs mit digitalen Daten. Sie umfasst die Auseinandersetzung mit digitalen Daten, angefangen bei ihrer Entstehung über die Prozesse, Instrumente und Infrastrukturen zu ihrer Verarbeitung, Analyse und Bereitstellung inklusive Publikation bis hin zu ihrer langfristigen Sicherung und Nachnutzung. Neben dem planvollen und kritischen Einsatz von Daten für verschiedene (interdisziplinäre) Kontexte ist die kritische Auseinandersetzung, d.h. das Verstehen, Analysieren und Bewerten von rechtlichen, technischen und organisatorischen Rahmenbedingungen, Anforderungen und Lösungen bedeutend. Dieses konzeptuelle Wissen ist darüber hinaus in die verschiedenen Domänen wie Forschung und Wissenschaft, Kultur, Gesellschaft und Wirtschaft übertragbar. ${ }^{15}$

Datenmanagement ist eine Kernkompetenz für die transparente und partizipative globale Forschung, wie zahlreiche Projekte und Initiativen zeigen. Im Kontext der Aus- und Weiterbildung kann diese Einschätzung für den Bereich der Studiengänge durch eine Marktstudie zumindest für Deutschland nicht bestätigen werden: Auf Basis von Daten der Hochschulrektorenkonferenz (HRK) wurde an der Fachhochschule Potsdam eine Analyse und Auswertung aller zum Thema Datenmanagement relevanten Studiengänge vorgenommen. Sie kommt zu dem Ergebnis, dass es derzeit kein vergleichbares Studienangebot in Deutschland mit der beschriebenen inhaltlichen Ausrichtung auf Datenmanagement gibt. ${ }^{16}$

Die Spezialisierung des hier vorgestellten Studiengangs auf wissenschaftspolitische, organisatorische, technische und rechtliche Aspekte im Bereich des Datenmanagements ist innovativ und stellt ein Desiderat besonders für die Qualifizierung im wissenschaftlichen Datenmanagement dar. Der Studiengang Digitales Datenmanagement ist der erste in Deutschland mit dem Schwerpunkt auf die

\footnotetext{
13. Vgl. z.B. Stifterverband: Data Literacy Education, https://www.stifterverband.org/ data-literacy-education (18.01.2019)

14. Vgl. z.B. Maxi Kindling and Peter Schirmbacher, ,, Die digitale Forschungswelt' als Gegenstand der Forschung," Information - Wissenschaft \& Praxis 64, nos. 2-3 (2013); Heike Neuroth et al., „Aktives Forschungsdatenmanagement," $A B I$ Technik 38, no. 1 (2018): 55-64.

15. Ausführlicher zu dieser Definition in Heike Neuroth et al., „Digitales Datenmanagement als neue Aufgabe für wissenschaftliche Bibliotheken," erscheint, Bibliothek Forschung und Praxis

16. Vgl. ibid.
} 
Vermittlung der Datenmanagement-Kompetenzen aus dem EDISON Framework und dem darauf aufbauenden EOSCpilot. Er wird eine informationswissenschaftliche Perspektive auf das Thema vermitteln und besonders auf die Domäne Wissenschaft fokussieren, wobei Kultur und Wirtschaft als Domänen ebenfalls berücksichtigt werden. Perspektivisch sollen letztere Domänen stärker ausgebaut und integriert werden.

Der Studiengang baut auf einer für Deutschland einzigartigen Kooperation zweier im Umfeld des Datenmanagements etablierter bibliotheks- und informationswissenschaftlicher Hochschulen auf. Die Verbindung bereits vorhandener Kompetenzen aus Forschung und Lehre von einer Forschungsuniversität mit einer anwendungsorientierten Fachhochschule vereinen die für das Profil des Studiengangs notwendigen inhaltlichen Voraussetzungen. An der Humboldt-Universität zu Berlin ist das Management von Forschungsdaten in den vergangenen Jahren zu einem einrichtungsübergreifenden Service ausgebaut worden. Die Initiative Forschungsdatenmanagement an der Humboldt-Universität wurde durch das IBI mit angestoßen und ist eine gemeinsame Initiative der Zentraleinrichtungen Computer- und Medienservice (CMS), Servicezentrum Forschung sowie Universitätsbibliothek und des Vizepräsidenten für Forschung. Am IBI wurde unter anderem das Drittmittelprojekt re3data.org - Registry of Research Data Repositories durchgeführt. Ein Zentrum für Digital Literacy, welches Fragen der Data Literacy umfasst und erforscht, ist am IBI im Aufbau begriffen. Das Lehrangebot im Bereich Forschungsdaten besteht bereits seit einigen Jahren. ${ }^{17}$ An der Fachhochschule Potsdam wurde das Lehrangebot sowohl im Bachelor als auch im Master in den letzten Jahren kontinuierlich in Richtung Datenmanagement und Forschungsdaten(management) ausgebaut. Die Berufungspolitik der letzten Jahre spiegelt diese Schwerpunktsetzung ebenfalls wieder: So erfolgte 2017 eine Neu-Berufung mit der Denomination Open Access/Open Data im Studiengang Bibliothekswissenschaft ${ }^{18}$, eine geplante Neuausrichtung im Studiengang Informations- und Datenmanagement ist mit einer neuen Denomination im Bereich Angewandte Datenwissenschaft in Vorbereitung. Darüber hinaus gibt es bereits zahlreiche, laufende Drittmittelprojekte, so zum Beispiel das DFG-geförderte Projekt „Research Data Management Organiser" $\left(\mathrm{RDMO}^{19}\right)$, das EU-Projekt PARTHENOS ${ }^{20}$ oder das jüngst bewilligte DFG-geförderte Projekt „Management Molekularer Daten im Research Data Life Cycle" (MaMoDaR), welches in Kooperation und unter Leitung des Robert Koch-Instituts im 2. Quartal 2019 starten wird.

\footnotetext{
17. Vgl. Kindling and Schirmbacher, ,, Die digitale Forschungswelt' als Gegenstand der Forschung."

18. https://www.fh-potsdam.de/informieren/aktuelles/news-detailansicht/artikel/ ellen-euler-tritt-professur-fuer-open-access-open-data-an/ (18.01.2019)

19. https://rdmorganiser.github.io/ (18.01.2019)

20. http://www.parthenos-project.eu/ (18.01.2019)
} 


\section{Der weiterbildende Masterstudiengang Digitales Datenmanagement (DDM)}

\section{Organisation des Studiengangs}

Der Studiengang wird voraussichtlich im Sommersemester 2020 mit 25-30 Studienanfängerinnen starten. ${ }^{21}$. Die Lehre wird dabei hälftig in Berlin und hälftig in Potsdam an den jeweiligen Instituten stattfinden. Die Dozierenden des Studiengangs setzen sich aus Mitarbeitenden beider Institute sowie fachlich ausgewiesener Lehrbeauftragter aus der Forschung und Wirtschaft zusammen.

Der Abschluss Master of Arts (M.A.) im Fach Digitales Datenmanagement wird nach erfolgreichem Studienabschluss gemeinsam von der Fachhochschule Potsdam und der Humboldt-Universität zu Berlin verliehen. Die Akkreditierung des Studienganges ist in Vorbereitung. Der Studiengang ist als weiterbildender Studiengang kostenpflichtig. Das Studienentgelt wird 1.975 Euro pro Semester zuzüglich der von der Hochschule erhobenen Semestergebühren (z.Z. 110 Euro) betragen.

Die Regelstudienzeit ist mit zwei Jahren (vier Semester) angesetzt und umfasst eine geplante Arbeitszeit von 3.000 Stunden, einschließlich der Erarbeitung einer Masterarbeit und deren Verteidigung. Der Studiengang arbeitet mit der Lehr- und Lernform des Blended Learnings, d.h. der Großteil des Studiums wird im Selbststudium verbracht, ein weiterer Anteil als Präsenzlehre. Die Präsenzlehre findet an vier zweitägigen Präsenztagen (jeweils Freitag und Samstag) im Semester statt, d.h. die Studierenden verbringen während der zwei Jahre insgesamt 32 Präsenztage an den Hochschulen. Alle Materialien, Studiendokumente sowie Kommunikationsforen für den Austausch mit den Dozierenden und miteinander werden über das Lernmanagementsystem Moodle bereitgestellt. Ein Studium ist berufsbegleitend möglich, vorausgesetzt Arbeitnehmer können an den Präsenztagen (die Termine werden im Vorfeld des Studiums mitgeteilt) teilnehmen.

\section{Struktur, Lern- und Qualifikationsziele des Studiengangs}

Der Studiengang wird grundsätzlich Kompetenzen der Forschungs- und Handlungsfelder im Datenmanagement vermitteln, die den formulierten Kompetenzgruppen gemäß des EDISON Framework

21. http://www.ddm-master.de/ (18.01.2019) 
und des EOSCpilot Reports D7.1 unter Berücksichtigung der nationalen (deutschen) und internationalen wissenschaftspolitischen, organisatorischen, technischen und rechtlichen Rahmenbedingungen entsprechen. Im Mittelpunkt steht - auch in Anlehnung an das oben ausgeführte Verständnis der Data Literacy - die Vermittlung von Fähigkeiten und Kompetenzen im analytischen wie praktischen Umgang mit Forschungs- und Informationsinfrastrukturen sowie der Ermittlung von Anforderungen und Lösungen für Informationssysteme, die das Datenmanagement unterstützen (wie beispielsweise digitale Repositorien). Die Domäne Wissenschaft und Forschung nimmt eine primäre Rolle ein, die Domänen Kultur und Wirtschaft sollen perspektivisch stärker einbezogen werden. Einen detaillierteren Überblick über das Studium bieten das Modulhandbuch sowie der Studienverlaufsplan. ${ }^{22}$ An dieser Stelle sollen die Struktur sowie beispielhaft einige wichtige Kompetenzen vorgestellt werden, die der Studiengang vermittelt.

Tabelle 1 zeigt eine vereinfachte Darstellung der Studienstruktur (Planungsstand: März 2019). Über vier Semester werden die Studierenden Module durchlaufen, die die Rahmenbedingungen, die Technologien sowie die Methoden des Datenmanagements sowohl theoretisch als auch angewandt vermitteln. In Projektarbeiten und Reallaboren, die begleitend zu den jeweiligen Themenaspekten durchgeführt werden, werden domänenspezifische Kenntnisse erworben bzw. vertieft und die einschlägige Berufspraxis der Studierenden mit den Studieninhalten integriert und kritisch reflektiert. Diese Komponenten dienen im Studiengang besonders dem Transfer von theoretischen Kenntnissen in die praktische Anwendung bzw. umgekehrt dem Transfer von praktischen Aktivitäten in konzeptuelle Modelle bzw. Rahmenwerke. Jedes Modul wird mit einer schriftlichen Ausarbeitung (Hausarbeit, Projektarbeit, Konzeptarbeit etc.) abgeschlossen. Ein letzter Pflichtbestandteil ist die Erarbeitung und Verteidigung der Masterarbeit. ${ }^{23}$ Im Modul Rahmenbedingungen werden grundlegende Konzepte des Datenmanagements und des Lebenszyklus von Daten insbesondere in der wissenschaftlichen Forschungslandschaft definiert. Institutionelle, rechtliche und politische Rahmenbedingungen von Forschungs- und Informationsinfrastrukturen werden beschrieben und sollen mit anderen Domänen in Beziehung gesetzt werden. Ein Modulkurs fokussiert auf Standards, z.B. CERIF $^{24}$ Common European Research Information Format) für Forschungsinformationssysteme,

22. http://www.ddm-master.de/index.php/studieninhalte/ (18.01.2019)

Diese Angaben gelten vorbehaltlich der Zustimmung der Gremien beider Hochschulen sowie der zuständigen Ministerien.

23. http://www.ddm-master.de/index.php/studieninhalte/ (18.01.2019)

Diese Angaben gelten vorbehaltlich der Zustimmung der Gremien beider Hochschulen sowie der zuständigen Ministerien.

24. euroCRIS. CERIF - the Common European Research Information Format. https://www.eurocris.org/ cerif/main-features-cerif (18.01.2019) 


Zeitraum Inhalte

1. Semester

2. Semester

3. Semester

4. Semester
Rahmenbedingungen des Datenmanagements

Technologien des Datenmanagements

Methoden des Datenmanagements

Masterabschlussprüfung (einschl. Masterarbeit)

Table 1: Geplante Struktur des weiterbildenden Masterstudiengangs Digitales Datenmanagement (Planungsstand: März 2019)

und Interoperabilitäts- und Metadatenanforderungen, z.B. die FAIR Data Principles. ${ }^{25}$

Das Modul Technologien des Datenmanagements fokussiert auf Internet- und Webtechnologien, Datenmanagementsysteme und Digitale Repositorien und führt in Algorithmen und Datenstrukturen ein. Studierende lernen eine Reihe von Technologie-Standards kennen, z.B. relationale Datenbankmodelle, XML-Datenbanken, TripleStores, NoSQL-Datenbanken im Datenbanken-Modulkurs, die je nach Interesse in einer Projektarbeit vertieft werden können.

Die wichtigsten Methoden des Methoden-Moduls sind neben der grundsätzlichen Diskussion von Anforderungen und Lösungen für das Forschungsdatenmanagement die Entwicklung von Datenmanagementplänen, (deskriptive) statistische Verfahren sowie Data Analytics, z.B. im Big Data Bereich und Datenvisualisierung.

Ausgehend vom Lebenszyklus von Forschungsdaten wurden im EOSCpilot D7.1 Fertigkeiten (Skills) aus drei vorherigen Projekten ${ }^{26}$ herangezogen und Kompetenzgebieten zugeordnet ${ }^{27}$, die nachfolgend anhand der Kompetenzgebiete „, plan and design“, „,integrate and analyse“ sowie „,appraise and preserve" dargestellt werden. Die Tabelle zeigt daneben die entsprechenden geplanten Lehrangebote im Studiengang Digitales Datenmanagement auf.

25. Mark D. Wilkinson et al., "The FAIR Guiding Principles for scientific data management and stewardship," Scientific Data 3 (2016): 18

26. EDISON Data Science Competence Framework: http://edison-project.eu/; RDA IG Education and Training in data handling: https://rd-alliance.org/groups/ education-and-training-handling-research-data.html; Data Information Literacy: http: //www. datainfolit.org/ (18.01.2019)

27. Whyte et al., Skills landscape analysis and competence model ab S. 60 
EOSC Compe- EOSCpilot D7.1 Kompetenzen tence Group

Plan and design Data management planning

Data model development

Database sepcification and design

Metadata specification

File format selection

Data repository requirements

$\begin{aligned} & \text { Integrate and } \\ & \text { analyse }\end{aligned}$
Data mining

Data versioning

Data transformation

Data processing and statistical analysis tools

Analysis workflows

Appraise and pre-

Data provenance serve management

Theoretische Grundlagen Datenmanagement und Data Literacy Forschungsdatenmanagement Datenmanagementpläne Open Access, Open Data, Open Science

Metadaten, Standards, Interoperabilität

Digitale Repositorien

Informationstechnische Grundlagen: Internet- und Webtechnologien

Informationstechnische Grundlagen: Datenmanagementsyteme

Einführung in Algorithmen und Datenstrukturen

Statistische Methoden in der Datenaufarbeitung- und Auswertung

Datenanalyse und Datenvisualisierung

Metadaten, Standards, Interoperabilität 
Data quality

Data review and appraisal

Preservation planning

File format migration

Data preservation
Forschungsdatenmanagement

Datenmanagementpläne

Table 2: Lehrangebote im Studiengang Digitales Datenmanagement

Ein wichtiger Bestandteil des Studiums ist die Verbindung zwischen Wissenschaft und Praxis, die in der Studienstruktur fest durch Projektarbeiten und Reallabore verankert ist. In Projektarbeiten können die Studierenden entweder ihre eigenen Praxiserfahrungen mit den Studieninhalten verknüpfen oder die vermittelten Inhalte in angewandte Fähigkeiten umsetzen, z.B. in einer Evaluation oder dem Vergleich von Ansätzen für Informationsinfrastrukturangebote, der Erstellung von Konzepten für eine reales Datenmanagement bzw. Datenarchivierung sowie der Implementierung eines Workflows für diese Aufgaben. Die Integration von praktischen Herausforderungen mit theoretisch fundierten und im Studium vermittelten Analysemethoden bzw. Implementierungswerkzeugen wird die unmittelbare Anwendbarkeit der Studieninhalte nicht nur für die Studierenden, sondern auch für die Arbeitgeber demonstrieren und dabei gleichzeitig die Lerneffektivität steigern. Der Studiengang wird so im Laufe der Zeit eine Reihe von Fallstudien erarbeiten, die dann wiederum in die Lehre einfließen werden.

\section{Zugangs- und Zulassungsvoraussetzungen für den Studiengang}

Für die Zulassung zum Studiengang ist ein berufsqualifizierender Hochschulabschluss im Umfang von 180 ECTS-Leistungspunkten Voraussetzung - dies entspricht einem Bachelorabschluss. Der Hochschulabschluss kann in jedweder Fachrichtung erfolgt sein. Dies entspricht der beruflichen Realität, in der viele Datenmanager aus anderen Disziplinen kommen und erst während der Berufspraxis oder in Praktika eine Affinität bzw. Expertise im Datenmanagement oder anderen datenintensiven Tätigkeiten aufgebaut haben.

Eine weitere Zulassungsvoraussetzung ist der Nachweis einer mindestens 12-monatigen den Studieninhalten zuträglich Berufserfahrung im Umfang einer Vollzeittätigkeit (einschließlich Praktika und 
wissenschaftliche Hilfskrafttätigkeiten), die kumulativ über die letzten fünf Jahre vor dem Bewerbungszeitpunkt erworben werden kann. Zwei Monate Berufserfahrung müssen konkret im Bereich (digitaler) Informations- und Datenwissenschaft bzw. datenintensiver Tätigkeiten nachgewiesen werden (siehe oben).

Neben den entsprechenden Nachweisen müssen Bewerberinnen ein Motivationsschreiben einreichen, dass die eigene wissenschaftliche und berufliche Laufbahn in den Kontext der Studienziele und erwarteten Kompetenzen stellt.

\section{Datenmanager, Data Librarian, Data Scientist oder Data Steward - Ein gefragtes Berufsbild}

Die Inhalte des Studienganges besitzen hohe Relevanz für die berufliche Praxis und sollen auch für höher dotierte Positionen qualifizieren. Breite Einsatzmöglichkeiten finden sich an Hochschulen, in Forschungseinrichtungen, Serviceeinrichtungen der Informationsinfrastruktur sowie Wirtschaftsunternehmen gleichermaßen.

Der Rat für Informationsinfrastrukturen (RflI) spricht von neuen Berufsbildern, „wie z. B. den (digitalen) Dokumentar, den Data Librarian, den Datenarchivar oder den Data Scientist mit Spezialisierung",28 die als Ergebnis aus Studiengängen für datenintensive Forschung hervorgehen. Die unterschiedlichen Benennungen des Berufsbildes kann man auf unterschiedliche institutionelle Kontexte (Bibliotheken, Archive, Informationsinfrastrukturen), aber auch auf stärkere Spezialisierungen (z.B. Datenanalyse, Datenarchivierung) zurückführen.

Der Studiengang greift die Bezeichnung Data Steward ${ }^{29}$ auf, um über die spezifischen Kontexte hinweg ein Berufsfeld zu definieren, welches einen Fokus auf die Generierung, Verarbeitung, Beschreibung, Repräsentation, Bereitstellung, Nutzung und Archivierung von Daten legt - also den Lebenszyklus von Daten organisatorisch und technologisch steuern und unterstützen kann. Diese datenintensiven Tätigkeiten werden nicht durch den Studiengang oder durch die Forderungen der (wissenschafts-)politischen Papiere definiert und gegründet, sie finden aktuell in vielen Organisationen statt. Der Studiengang spricht daher einerseits diejenigen Zielgruppen an, die durch Spezialisierung, Training-on-the-Job oder Aufgabenumverteilung bzw. -restrukturierung schon längst diese

28. RFII - Rat für Informationsinfrastrukturen, Leistung aus Vielfalt, 50.

29. Der Begriff wird u.a. weitläufig verwendet und definiert in: Simon Hodson et al., „Turning FAIR data into reality: interim report from the European Commission Expert Group on FAIR data," 2018, 
Tätigkeiten ausführen, und andererseits diejenigen interessierten Studierenden, die sich in das Feld neu einarbeiten möchten. Dabei wird fokussiert auf die Domäne Wissenschaft und Forschung (dies kann allerdings auch in nicht-öffentlichen Einrichtungen geschehen). Die Domänen Kultur und Wirtschaft sollen perspektivisch auch verstärkt angesprochen werden. Konkret sollen folgende Zielgruppen mit dem Studiengang angesprochen werden:

- Interessentinnen an Datenmanagement, -prozessierung, -analyse und -bereitstellung in den Domänen Forschung, Kultur und Wirtschaft

○ Forschungsreferentinnen

- Mitarbeiterinnen im Forschungsservice und Wissenschaftsmanagement

○ Daten-Produzenten (z. B. Digitalisierungslabore, Verwaltungen, Forschungseinrichtungen)

○ Daten-Verarbeiterinnen im öffentlichen und kulturellen und wirtschaftlichen Sektor

- Mitarbeiterinnen von Serviceeinrichtungen der Informationsinfrastruktur (Bibliotheken, Museen, Archive, Dokumentationseinrichtungen, sonstige wissenschaftliche Informationseinrichtungen)

- Mitarbeiterinnen in kleinen, mittleren und großen Unternehmen im Daten-Service-Bereich (Verlage, Pharma-Unternehmen etc.)

Das Ziel des Studiengangs ist es, Kompetenzen für datenintensive Tätigkeiten, die von vielen ad-hoc und bedarfsbezogen in der eigenen Berufspraxis aufgebaut wurden, in ein wissenschaftlich strukturiertes Rahmenwerk bzw. in konzeptuelle Modelle zu integrieren, so dass Zusammenhänge über den eigenen institutionellen oder organisatorischen Kontext hinaus erkannt und angewandt werden können. Absolventinnen sollen befähigt werden, Trends und Anwendungen aus spartenfremden Fachgebieten schneller zu erkennen und sich neue Anwendungsbereiche zu erobern. Langfristig kann der Studiengang so zu einer Konvergenz innerhalb des Berufsfeldes beitragen.

Eine Mischung von Studierenden aus unterschiedlichen institutionellen und organisatorischen Kontexten, aus verschiedenen Fachgebieten und unterschiedlichen Erfahrungsebenen ist eine Herausforderung für jede Qualifizierungsmaßnahme. Der Studiengang plant, die Heterogenität der Zielgruppen konkret in die Lehre zu integrieren, indem Fallstudien und Probleme von Studierenden eingebracht werden und in gemischten Gruppen in den Präsenzlehrtagen aufbereitet werden. Studierende sollen durch intensives und durch die Studienorganisation unterstütztes Networking 
bzw. Erfahrungsaustausche nicht nur von den Dozierenden und bereitgestellten Lehrmaterialien lernen, sondern auch voneinander.

\section{Zusammenfassung: Herausbildung eines neuen Berufsbildes}

Es sind die "Köpfe“, die durch Integration von Daten, Informationen und Wissen aus heterogenen Quellen und über Domänengrenzen hinweg für die Entstehung neuen wissenschaftlichen Wissens und damit für die Wertschöpfung aus den Daten sorgen. ${ }^{30}$

Die diversifizierte Informationslandschaft erfordert einen hohen Grad an Flexibilität, Dynamik und Spezialisierung an die Informations- und Datenprofessionen, die den oben genannten Anforderungen genüge tragen müssen. Der Studiengang Digitales Datenmanagement zielt darauf ab, Absolventinnen auf diese Aufgaben vorzubereiten. Ein besonderes Ziel des neuen weiterbildenden Masterstudienganges ist es, die Fähigkeiten und Anforderungen an Datenmanager bzw. Data Stewards nicht nur zu vermitteln, sondern auch das Berufsbild - mag man es Data Librarian, Data Curator, Data Manager oder Data Steward nennen - in dieser Landschaft zu festigen und zu verankern.

Durch die Standardisierung eines Curriculums und durch die Zertifizierung im Masterabschluss sollen Informations- und Datenspezialisten in diesem Gebiet eine Grundlage erhalten, ihr Berufsbild nicht nur zu definieren, sondern auch zu repräsentieren. Zukünftigen Absolventinnen dieses und ähnlicher Studiengänge bietet der Studiengang eine Plattform, ihre speziellen Fähigkeiten in einer Berufsqualifikation abzubilden. Für Informationsinfrastruktureinrichtungen und andere potenzielle Arbeitgeber bietet der Abschluss eine Basis, um qualifizierte Datenmanager zu identifizieren und aufgabengerecht einzusetzen.

Die im Hochschulabschluss zertifizierte Berufsqualifikation wird auch in Tarifdiskussionen für Berufsgruppen eine Rolle spielen. Dem Ruf der Politik nach verbesserter Hochschulbildung und Ausbildung für die Digitalisierung und Datenrevolution wird gefolgt und die ersten Schritte sind getan. Für die Etablierung des Berufsstandes müssen Politik und Profession weiter zusammenarbeiten, um diese Spezialisierung auch in tarifrechtlichen Bestimmungen angemessen zu verankern. Absolventinnen aber auch Arbeitgebern wird eine gemeinsame Diskussionsgrundlage und wissenschaftliche Basis gegeben, um diesen Anpassungsprozess in den verschiedensten Einrichtungen bzw. Unternehmen zu begleiten.

30. RFII - Rat für Informationsinfrastrukturen, Leistung aus Vielfalt, 3. 


\section{Literaturverzeichnis}

Edison Project. EDISON Data Science Framework: Part 1. Data Science Competence Framework (CF DS). Release 2, 2017. http://edison-project.eu/sites/edison-project.eu/ files/filefield\%5C_paths/edison\%5C_cf-ds-release2-v08\%5C_0.pdf.

High Level Expert Group on the European Open Science Cloud. Realising the European Open Science Cloud. 2016. doi:10.2777/940154.

Hochschulrektorenkonferenz. Gemeinsame Erklärung von Hochschulleitungen, die am Workshop der HRK zum Forschungsdatenmanagement am 16.12.2016 teilgenommen haben. 2016. https: //www.hrk.de/fileadmin/redaktion/hrk/02-Dokumente/Erklaerung\%5C_Workshop\% 5C_Forschungsdatenmanagement\%5C_16122016.pdf.

Hodson, Simon, Sarah Jones, Sandra Collins, Françoise Genova, Natalie Harrower, Leif Laaksonen, Daniel Mietchen, Rūta Petrauskaité und Peter Wittenburg. „Turning FAIR data into reality: interim report from the European Commission Expert Group on FAIR data", 2018. doi:10 . 5281 /zenodo. 1285272.

Kindling, Maxi, und Peter Schirmbacher. „, Die digitale Forschungswelt' als Gegenstand der Forschung". Information - Wissenschaft \& Praxis 64, Nrn. 2-3 (2013). doi:10.1515/iwp-20130017.

Neuroth, Heike, Claudia Engelhardt, Jochen Klar, Jens Ludwig und Harry Enke. „Aktives Forschungsdatenmanagement". ABI Technik 38, Nr. 1 (2018): 55-64. doi:10.1515/abitech2018-0008.

Neuroth, Heike, Laura Rothfritz, Vivien Petras und Maxi Kindling. „Digitales Datenmanagement als neue Aufgabe für wissenschaftliche Bibliotheken". Erscheint, Bibliothek Forschung und Praxis.

RFII - Rat für Informationsinfrastrukturen. Leistung aus Vielfalt: Empfehlungen zu Strukturen, Prozessen und Finanzierung des Forschungsdatenmanagements in Deutschland. Göttingen, 2016. http://www.rfii.de/?p=1998.

Whyte, Angus, Kevin Ashley, Rahul Thorat und Eileen Kuehn. Skills landscape analysis and competence model 7.1. EOSCpilot, 2017. Besucht am 19. Dezember 2018. https://eoscpilot. eu/sites/default/files/eoscpilot-d7.3.pdf. 
Whyte, Angus, Jerry de Vries, Rahul Thorat, Eileen Kuehn, Gergeley Sipos, Valentino Cavalli, Vasso Kalaitzi und Kevin Ashley. Skills and Capability Framework Deliverable 7.3. EOSCpilot, 2018. Besucht am 19. Dezember 2018. https://eoscpilot.eu/sites/default/files/ eoscpilot-d7.3.pdf.

Wilkinson, Mark D., Michel Dumontier, IJsbrand Jan Aalbersberg, Gabrielle Appleton, Myles Axton, Arie Baak, Niklas Blomberg u. a. „The FAIR Guiding Principles for scientific data management and stewardship". Scientific Data 3 (2016): 160018. doi:10.1038/sdata. 2016. 18. 